\section{Call to veto Brazil's forest-code revisions}

At last month's COP17 climatechange negotiations in Durban, South Africa, Brazilian president Dilma Rousseff called for renewal of the Kyoto Protocol's binding targets for reducing greenhousegas emissions. We look to her to reaffirm Brazil's commitment to sustainable development by vetoing the proposed changes to the country's Forest Code when it comes before the Brazilian National Assembly for ratification in March.

The Senate has already approved revisions to the code, which would provide amnesty for previously illegal forest clearance and undermine efforts to limit deforestation.

Brazil should be setting an example as a green economy, particularly as it will be hosting the Rio+20 conference this year - the twentieth anniversary of the 1992 United Nations Conference on Environment and Development.

The adverse environmental effects that would be generated by the Forest Code revisions threaten to be made worse by the country's poor record for enforcing environmental law. For example, appeals against fines for environmental crimes are almost always successful (see go.nature. $\mathrm{com} / 2 \mathrm{xvs} 2 \mathrm{o}$ ), amounting to nearly US $\$ 8$ billion in unpaid penalties between 2005 and 2010 .

Brazil's forestry regulations must be tailored to ensure the protection of forests alongside sustainable development. The new Forest Code would do neither.

Alison G. Nazareno Federal University of Santa Catarina, Florianópolis, Brazil. alison_nazareno@yahoo.com.br

\section{Mauritius is putting conservation at risk}

A government policy for the national parks of Mauritius is threatening important research into conservation and undermining the ownership and sustainability of conservation projects. Appeals to modify this policy have remained unanswered.

Mauritius is known for its conservation successes including that for the Mauritian kestrel, Falco punctatus thanks to the development of innovative techniques. Its national parks provide ideal terrain for conservation research because they harbour most of the country's highly threatened biodiversity and provide a variety of experimental settings for study.

The new policy makes it difficult for biologists and students from the local university to access field camps, restricting their fieldwork to office hours on weekdays. Fieldwork outside these periods is rarely allowed: applications typically take at least three months to process, and payment to the National Parks and Conservation Service is necessary if the request is successful.

The Mauritian government's policy is insensitive to the temporal expression of biological phenomena. Its justification of concern for biologists' safety is weak, given that no significant security scares have ever been recorded. Worse, many foreign biologists are allowed to work at the same sites, unimpeded by the restrictions facing local academics.

The Mauritian authorities should note that the local university has been spearheading capacity-building to support native conservation efforts since the 1990s, with more than 50 research projects completed by its graduate and undergraduate students. With the help of the international community, the government should be persuaded to return to a stance that is less harmful to conservation.

F. B. Vincent Florens University of Mauritius, Réduit, Mauritius. vin.florens@uom.ac.mu

\section{Phoenix first to see silt grains on Mars}

The successful launch of NASA's

Mars Science Laboratory, or Curiosity, offers a chance to correct a misconception about its imaging systems. You say that these have sufficient magnification and resolution "to see, for the first time, tiny grains of silt ... particles that would bear the history of billions of years of erosion from wind and water"

(Nature 479, 446; 2011).

Curiosity's imager will be the first to see silt-sized particles in situ, on the undisturbed surfaces of rocks and soils. But bragging rights for the first images of silt grains (4-62.5 micrometres in diameter) and even some claysized particles (smaller than 4 micrometres in diameter) go to the Phoenix Mars Lander, which accomplished the feat in 2008 (P. H. Smith et al. Science 325, 58-61; 2009).

Soil samples from trenches on the periglacial landforms surrounding Phoenix were imaged by an optical microscope in the Microscopy, Electrochemistry, and Conductivity Analyzer (MECA), which returned hundreds of colour images of grains as fine as 4 micrometres in diameter (W. Goetz et al. J. Geophys. Res. 115, E00E22; 2010). Imaging grains from diverse substrates enables the microscopic characterization of grain magnetic properties. The MECA atomic-force microscope returned threedimensional images of particles as small as 0.1 micrometres (M. A. Velbel and A. I. Losiak J. Sed. Res. 80, 771-780; 2010).

When it lands, Curiosity will comprise the most capable suite of instruments delivered to the surface of another planet. But Phoenix has already given us a close look at Mars. Michael Velbel Michigan State University, East Lansing, Michigan, USA. velbel@msu.edu

\section{Science and politics need more empathy}

Some important pointers for improving communication between scientists and politicians (Nature 480, 153; 2011) emerged from a meeting last year between the two groups, organized by the International Risk Governance Council.

Support for fundamental research is essential, but scientists shouldn't be tempted to overstate their claims. Specifically, requests for funding for basic research into a particular question shouldn't imply that the research will definitely provide the answer. When basic questions lead to technological advances, these typically emerge in another, unexpected area - take quantum mechanics and the transistor, for example, or the study of gas conductivity that led to the discovery of X-rays.

The best bets for answering immediate, focused questions are likely to be the development of existing technologies and the novel juxtaposition of established ideas from different areas. Governments should therefore put more faith in interdisciplinary scientists who have the vision and ability to bring apparently disparate fields together, rather than turning to subject specialists, who often have their own agendas.

For their part, scientists must recognize and respect the need of politicians to win votes. Without power, politicians can't carry through a long-term, scientifically based policy. Scientists who promote these policies will fare better if they can identify and suggest short-term, intermediate benefits.

Len Fisher University of Bristol, Bristol, UK.

len.fisher@bristol.ac.uk

CONTRIBUTIONS

Please consult the author guidelines for submitting Correspondence at go.nature.com/cmchno. 